\title{
Perfil obstétrico de gestantes atendidas em pré-natal em centro de saúde da família
}

\author{
Obstetric profile of pregnant women attended \\ in prenatal care at the family health center
}

LEONARdo SALES Ribeiro SilVA Ana Neiline Cavalcante ${ }^{I}$ Maria Auxiliadora Silva OLIVEIRA ${ }^{\mathrm{I}}$

${ }^{\mathrm{I}}$ Centro Universitário Instituto Superior de Teologia Aplicada (INTA-UNINTA). Sobral/CE - Brasil

Fomento: Bolsa de Iniciação Científica do Centro Universitário Inta - UNINTA

\begin{abstract}
Resumo Objetivou-se traçar o perfil obstétrico de gestantes assistidas em um Centro de Saúde da Família no município de Sobral/CE. Trata-se de uma pesquisa retrospectiva, quantitativa e descritiva, tendo como fonte de dados os prontuários de gestantes atendidas nos anos de 2016 e 2017. Foi analisado um total de 64 prontuários, sendo coletada a faixa etária materna, o tipo de parto, o número de consultas pré-natal realizadas pela gestante, duração da gestação (em semanas), nível de escolaridade e estado civil. Os resultados revelam que a menor idade registrada foi de 12 anos, tendo o maior percentual na faixa dos 20 aos 30 anos de idade. No nível de escolaridade das gestantes, o valor predominante do grau de instrução foi no nível médio (71,87\%). Percebe-se no resultado referente ao estado civil das gestantes que os maiores percentuais estiveram entre o estado civil casada $(42,18 \%)$ e união estável $(35,93 \%)$. Em relação ao tipo de parto, pôde-se constatar que o parto cesáreo foi de $62,5 \%$. Sobre o número de consultas pré-natal, grande parte realizou sete ou mais consultas $(84,3 \%)$. Para a duração da gestação, a maioria $(71,9 \%)$ esteve entre 37 a 41 semanas, sendo considerada a termo. O perfil das gestantes atendidas no Centro de Saúde da Família do bairro Dom Expedito Lopes é de mulheres jovens, com baixa escolaridade, casadas e submetidas a parto cesáreo. Essas informações mostram a importância de campanhas educativas de orientação e convencimento, quanto às vantagens do parto normal.
\end{abstract}

Palavras-chave: Idade materna. Tipo de parto. Pré-natal.

ABstractThe objective of this study was to trace the obstetric profile of pregnant women attending a Family Health Center in the municipality of Sobral/CE. This is a retrospective, quantitative and descriptive study, having as a data source the records of pregnant women attended in the years 2016 and 2017. A total of 64 medical records were analyzed and the maternal age group, type of delivery, the number of prenatal consultations performed by the pregnant woman, duration of gestation (in weeks), level of schooling and marital status. The results show that the lowest recorded age was 12 years, with the highest percentage in the range of 20 to 30 years. In the educational level of pregnant women, the predominant educational level was the average level $(71.87 \%)$. It can be seen in the result concerning the marital status of the pregnant women that the highest percentages were between the marital status (42.18\%) and stable union (35.93\%). Regarding the type of delivery, it can be seen that the 
cesarean, it was the $62.5 \%$. About the number of prenatal consultations, the majority performed seven or more consultations (84.3\%). For the duration of gestation, the majority $(71.9 \%)$ was between 37 to 41 weeks, being considered at term. The profile of pregnant women attending the Family Health Center in the neighborhood of Dom Expedito Lopes is of young women with low schooling, married and undergoing cesarean delivery. This information shows the importance of educational guidance and convincing campaigns about the advantages of normal birth.

Keywords: Maternal age. Type of delivery. Prenatal.

\section{INTRODUÇÃO}

A gestação é um período de extrema vulnerabilidade para a mulher, sendo caracterizado por uma série de transformações físicas, psíquicas e emocionais. ${ }^{1}$

Vale destacar nessa fase, a complexidade das mudanças provocadas pela vinda de um novo ser, sendo que não se restringem apenas às variáveis psicobiológicas, mas também a fatores socioeconômicos fundamentais nesse processo de mudança. Verifica-se que o nível de instrução, o acesso ao serviço de saúde, a classe econômica, a vida conjugal, o meio em que está inserida, a carga cultural, a renda familiar, entre outros, causam impactos de naturezas diversas. ${ }^{2}$

A assistência pré-natal tem como objetivo prestar assistência humanizada, acolhendo a mulher desde o início da sua gestação, visando à promoção da saúde da gestante e do feto; além de oferecer exames clínicos e laboratoriais durante as consultas de prénatal; fazer orientações, identificar situações de risco e agir precocemente, realizando intervenções oportunas, garantindo o fim da gestação com o nascimento de uma criança saudável e o bem-estar materno. ${ }^{3}$

A perspectiva de análise ancorada nos determinantes sociais, culturais e políticos da saúde tem permitido compreender o mecanismo que subjaz, como substrato, às "causas das causas" dos problemas de saúde, assim como, identificar os caminhos ou processos da produção de inequidades relativas à maternidade. As evidências mostram que a saúde reprodutiva, neonatal, mental e familiar se deteriora quando as mulheres têm empregos precários, falta de cobertura social durante a gravidez, moradia informal e insegura, baixa escolaridade, parceiro ausente do lar, risco psicossocial associado a apoio familiar insuficiente, sintomas depressivos, violência de gênero, abuso de substâncias e conflitos com a maternidade. ${ }^{4}$

O conhecimento sobre as características obstétricas de gestantes em determinado local e período é de fundamental importância, para possibilitar a elaboração de indicadores demográficos e epidemiológicos que podem representar a realidade da comunidade para o planejamento e implementação de estratégias efetivas de melhorias das condições de saúde. ${ }^{5}$

Sabe-se que, durante a gestação, o pré-natal é uma oportunidade para os profissionais identificar o perfil socioeconômico e gineco-obstétrico das gestantes, para conhecer os riscos aos quais a mulher está exposta durante a gestação e desenvolver as atividades de acompanhamento e promoção de uma gestação saudável. ${ }^{6}$

Sendo assim, o presente estudo tem como objetivo principal caracterizar o perfil 
obstétrico e sociodemográfico de gestantes que realizaram seu pré-natal no Centro de Saúde da Família do bairro Dom Expedito Lopes, na cidade de Sobral/CE.

\section{Metodologia}

A pesquisa foi realizada no Centro de Saúde da Família do bairro Dom Expedito Lopes, situado no município de Sobral/CE.

Trata-se de um estudo de caráter exploratório, quantitativo, descritivo, retrospectivo e com análise documental.

As participantes da pesquisa foram as gestantes $(n=64)$ atendidas no referido Centro de Saúde da Família, cujas fichas de acompanhamento e/ou prontuários datassem do ano de 2016 e 2017. Foram excluídas as fichas e/ou prontuários de anos diferentes desse tempo preestabelecido. Não foram excluídas, dentro desse período estabelecido, mulheres com características sociodemográficas e obstétricas com especificidades.

As variáveis analisadas foram aquelas que permitissem traçar um perfil obstétrico e sociodemográfico: idade materna, escolaridade, estado civil, tipo de parto, número de consulta pré-natal e idade gestacional. A renda salarial não estava disponível nos prontuários analisados, portanto, não fizeram parte desse perfil.

Os dados foram coletados a partir das fichas/prontuários de acompanhamento das gestantes que realizaram seu pré-natal no referido centro de saúde. Os prontuários utilizados encontravam-se arquivados no Serviço de Arquivo Médico e Estatística (SAME). Os dados foram analisados em $E x$ - cel e foram confeccionadas tabelas contendo frequências absolutas e relativas.

$\mathrm{O}$ presente trabalho foi submetido e aprovado pelo comitê de ética local, tendo sido aprovado com o número de protocolo 1.878.614.

\section{Resultados}

A Tabela 1 mostra a distribuição da faixa etária das gestantes. Pode-se perceber que a faixa etária predominante esteve entre as idades de 21 a 30 anos nos anos investigados.

Tabela 1 - Distribuição da faixa etária de gestantes atendidas no Centro de Saúde da Família do bairro Dom Expedito Lopes situado no município de Sobral/CE (2016 e 2017).

\begin{tabular}{lcc}
\hline Idade materna & $\mathrm{n}$ & $\%$ \\
\hline De $12-20$ & 13 & 20,3125 \\
De 21-30 & 37 & 57,8125 \\
De $31-40$ & 12 & 18,75 \\
41 & 02 & 3,125 \\
\hline Total & 64 & 100 \\
\hline
\end{tabular}

Fonte: Serviço de Arquivo Médico e Estatística (SAME).

Na Tabela 2, estão os resultados referentes ao nível de escolaridade das gestantes. Como pode ser observado, o valor predominante do grau de instrução é no nível médio $(71,87 \%)$.

Percebe-se no resultado referente ao estado civil das gestantes (Tabela 3) que os maiores percentuais estiveram entre o estado civil casada e união estável. 
Tabela 2 - Nível de escolaridade das gestantes atendidas no Centro de Saúde da Família do bairro Dom Expedito Lopes situado no município de Sobral/CE (2016 e 2017).

\begin{tabular}{l|c|c}
\hline Grau de instrução & $\mathbf{n}$ & \% \\
\hline Analfabeto & 02 & 3,125 \\
Fundamental & 15 & 23,4375 \\
Médio & 46 & 71,875 \\
Superior & 01 & 1,5625 \\
\hline Total & 64 & 100 \\
\hline
\end{tabular}

Serviço de Arquivo Médico e Estatística (SAME).

Tabela 3 - Estado civil das gestantes atendidas no Centro de Saúde da Família do bairro Dom Expedito Lopes, situado no município de Sobral/CE (2016 e 2017).

\begin{tabular}{l|c|c}
\hline Estado civil & n & \% \\
\hline Casada & 27 & 42,1875 \\
União estável & 23 & 35,9375 \\
Solteira & 14 & 21,875 \\
\hline Total & 64 & 100 \\
\hline
\end{tabular}

Serviço de Arquivo Médico e Estatística (SAME).

Como pode ser observado na Tabela 4, os resultados referentes ao tipo de parto realizados nas gestantes atendidas, predominou o parto cesáreo.

$\mathrm{Na}$ Tabela 5 consta o resultado referente ao número de consultas pré-natal realizadas pelas gestantes. Percebe-se que os maiores percentuais estiveram dentro das sete consultas ou mais realizadas.
Tabela 4 - Tipo de parto em gestantes atendidas no Centro de Saúde da Família do bairro Dom Expedito Lopes, situado no município de Sobral/CE (2016 e 2017)

\begin{tabular}{l|c|c}
\hline Tipo de parto & N & \% \\
\hline Cesáreo & 40 & 62,5 \\
Vaginal & 24 & 37,5 \\
\hline Total & 64 & 100 \\
\hline
\end{tabular}

Serviço de Arquivo Médico e Estatística (SAME).

Tabela 5 - Número de consultas pré-natal realizadas em gestantes atendidas no Centro de Saúde da Família do bairro Dom Expedito Lopes, situado no município de Sobral/CE (2016 e 2017).

\begin{tabular}{l|c|c}
\hline Número de consultas & $\mathbf{n}$ & \% \\
\hline $1-3$ & 00 & 00 \\
$4-6$ & 10 & 15,625 \\
$\geq 7$ & 54 & 84,375 \\
\hline Total & 64 & 100 \\
\hline
\end{tabular}

Serviço de Arquivo Médico e Estatística (SAME).

Observa-se, na Tabela 6, que o maior percentual referente à idade gestacional esteve dentro da faixa de 37 a 41 semanas.

Tabela 6 - Idade gestacional das gestantes atendidas no Centro de Saúde da Família do bairro Dom Expedito Lopes, situado no município de Sobral/CE (2016 e 2017).

\begin{tabular}{l|c|c}
\hline Idade gestacional (semanas) & $\mathbf{n}$ & $\mathbf{\%}$ \\
\hline $22-27$ & 01 & 1,5625 \\
$28-36$ & 13 & 20,3125 \\
$37-41$ & 46 & 71,875 \\
$\geq 42$ & 04 & 6,25 \\
\hline Total & 64 & 100 \\
\hline
\end{tabular}

Serviço de Arquivo Médico e Estatística (SAME). 


\section{Discussão}

É indiscutível a importância do prénatal como item de proteção para a mãe e o bebê. Existe forte associação entre cuidados pré-natais adequados e melhores resultados perinatais, seja em mulheres adolescentes ou em idade mais avançada. ${ }^{7}$

Os resultados evidenciados no estudo mostram a grande porcentagem $(57,81 \%)$ das gestantes entre a faixa etária de 21 a 30 anos (Tabela 1). A idade materna menor que 17 e maior que 35 anos representa um fator de risco importante na gravidez. ${ }^{8}$ Em um estudo realizado no interior de Minas Gerais, os autores encontraram gestantes predominando na faixa etária de 20 a 29 anos, resultados semelhantes aos encontrados na presente pesquisa. ${ }^{3}$

A adolescência é a fase de transição entre a infância e a idade adulta, e é definida pela Organização Mundial de Saúde como aquela compreendida entre 10 e 20 anos de idade incompletos, diferenciando-se da adolescência inicial (10- 14 anos) e a adolescência final (15-20 anos). ${ }^{9}$ Aproximadamente $20 \%$ das gestantes atendidas apresentaram idade entre 12 a 20 anos.

Para o Ministério da Saúde, o fenômeno da maternidade na adolescência é considerado de alto risco devido às complicações biológicas e sociais do binômio mãe e filho. As adolescentes com menos de 14 anos de idade têm maior risco de morrer durante a gravidez e seus filhos tendem a nascer com baixo peso e prematuros. $\mathrm{O}$ acompanhamento em qualquer gravidez é essencial para a saúde materna e fetal. E para tal, faz-se necessário a realização do pré-natal, que consiste em assistência médica e de enfermagem no de- correr da gravidez, com o objetivo de evitar problemas para a mãe e para a criança nesse período e no momento do parto. ${ }^{10}$

Muitas vezes a jovem gestante adia em procurar assistência médica por medo da reação da família ou falta de informação, o que compromete os resultados perinatais, assim como nas parturientes acima de 40 anos de idade, que têm relação a fatores prováveis como a hipertensão arterial, ruptura prévia de placenta, infecções urinárias e cardiopatias. ${ }^{7}$

No que diz respeito ao nível de escolaridade (Tabela 2), foi encontrado que o maior percentual $(71,87 \%)$ tem nível médio, o que implica dizer que existe informação sobre prevenção e concepção e se concluir que se tem o mínimo de informação acerca do assunto.

A educação é de extrema importância para a saúde da população, pois uma das motivações que levam as pessoas a procurar o serviço de saúde vem do grau de conhecimento que ela tem, e do seu grau de instrução. ${ }^{3}$

Com relação ao estado civil (Tabela 3), os maiores percentuais estiveram entre casada $(42,18 \%)$ e união estável $(35,93 \%)$. O predomínio de mães com apoio de um companheiro é considerado favorável, uma vez que a situação conjugal segura traz melhorias quanto à situação psicológica e estabilidade econômica. ${ }^{8}$ Esses resultados corroboram com aqueles encontrados por outros pesquisadores em um centro de saúde da família no norte de Minas Gerais, onde prevaleceu a situação de mulheres que viviam com seus companheiros $(84,6 \%){ }^{6}$

As gestantes que possuem vínculo afetivo com seus companheiros dividem os 
saberes sobre a gestação e juntos passam a conhecer a assistência prestada durante o pré-natal. Dessa forma, no decorrer do atendimento é necessário que se valorize tanto as opiniões do pai, quanto as da gestante, pois ambos têm um papel importante no pré-natal. ${ }^{11}$

O fato de haver jovens grávidas sem companheiros revela que, em muitos casos, o pai da criança pode abandonar a parceira por medo e insegurança, ou então, a gravidez pode ser decorrente de um relacionamento passageiro, não sendo impossível que o pai da criança, inclusive, desconheça a gravidez. De qualquer modo, parece mais fácil para o homem se afastar da situação. Com relação às jovens que permaneceram com seus parceiros, não se descarta que isso esteja ocorrendo por obrigação (social, familiar, religiosa), por um maior amadurecimento dos parceiros e das jovens, ou ainda, pela existência de um sentimento de amor e comprometimento entre eles. De qualquer forma, tal condição é positiva, pois o pai do bebê pode tornar-se uma importante fonte de apoio para as gestantes. ${ }^{12}$

O Brasil é o $2^{\circ}$. país no mundo em percentual de cesarianas. Enquanto a OMS estabelece em até $15 \%$ a proporção de partos por cesariana, no Brasil esse percentual é de $57 \% .{ }^{13}$ Elas representam $40 \%$ dos partos realizados na rede pública de saúde. Já na rede particular, chegam a $84 \%$ dos partos. ${ }^{14}$ $\mathrm{O}$ que pode comprovar-se por meio do resultado (Tabela 4) apresentado na pesquisa que mostra o parto cesáreo com maior percentil, $62,5 \%$.

Destaca-se, ainda, que o parto operatório aumenta o risco de morbidade e mortalidade maternas e perinatal. ${ }^{15}$ Outro estudo realizado com base em dados do Sistema de Nascidos Vivos, de 2000 até 2012, mostra que o principal público da cesariana no Brasil não são as mulheres em situação de maior risco. O pesquisador agrupou as mulheres de acordo com a quantidade de fatores de risco a que estavam sujeitas: baixa escolaridade, menos de 20 anos de idade, acesso a menos de quatro consultas de pré-natal, entre outros. Paradoxalmente, a maior frequência de cesarianas foi encontrada em gestantes sem nenhum fator de risco: $75,3 \%$ delas tiveram seus filhos por meio de cesarianas. Já entre as gestantes com quatro ou mais fatores de risco, apenas $30,7 \%$ foram submetidas à cirurgia. ${ }^{14}$

O período perinatal é uma fase onde se deve acompanhar a qualidade de saúde da mulher e do neonato, uma vez que muitas ações podem ser desenvolvidas nesse período, com o objetivo de evitar complicações durante a gestação e após o parto. O pré-natal deve ser iniciado imediatamente após a descoberta da gravidez, de forma universal e periódica, integrando ações preventivas e curativas com no mínimo seis consultas. A assistência pré-natal é importante para a relação mãe-filho. Observa-se que, no Brasil, a cobertura da assistência pré-natal é baixa, embora tenha sofrido desdobramentos nos últimos anos, indiferenças nesse tipo de assistência ainda prosseguem. ${ }^{16}$

Uma atenção pré-natal de qualidade é capaz de diminuir a morbidade e a mortalidade materno-infantil, uma vez que a identificação do risco gestacional pelo profissional permite a orientação e os encaminhamentos adequados em cada momento da gravidez. ${ }^{17}$ Em um estudo no interior de Minas Gerais, os autores verificaram que a grande maioria 
das gestantes havia realizado mais de seis consultas pré-natal. ${ }^{3}$ Esses achados corroboram com a presente pesquisa, conforme pode ser visualizado na Tabela 5 .

O número mínimo preconizado pelo Ministério da Saúde para todas as gestantes é de seis consultas, com início o mais precocemente possível, sendo assim distribuídas: uma no $1^{\circ}$. trimestre (até a $12^{\mathrm{a}}$ semana), duas no $2^{\circ}$. trimestre e três no $3^{\circ}$. trimestre; considerando o risco perinatal e as intercorrências clínico-obstétricas, mais comuns no $3^{\circ}$. trimestre, é imprescindível que sejam realizadas consultas até a $40^{\text {a }}$ semana de gestação; gestantes cujo trabalho de parto não se inicie até a $41^{\text {a }}$ devem ser encaminhadas à maternidade de referência para a interrupção da gravidez. ${ }^{18}$ De acordo com os dados coletados, a maioria das gestantes esteve presente nas sete consultas ou mais realizadas (Tabela 5).

O acompanhamento pré-natal não deve englobar somente os procedimentos clínicos, mas também os educativos, pois estes orientam a mulher sobre a evolução da gestação. A gestante não deve aceitar somente o que lhe é oferecido, mas também buscar o que é de seu direito, como o conhecimento adequado sobre seus problemas de saúde durante a gestação. ${ }^{19}$

Quanto às orientações, é certo que uma gama de temas deveria ser abordada durante o pré-natal, como promoção de comportamentos saudáveis, preparação e estímulo do parto normal e do aleitamento materno e informações sobre sinais de alerta. A assistência pré-natal, no Brasil, alcançou cobertura praticamente universal, mas persistem desigualdades no acesso a um cuidado adequado, que teria potencial de reverter os indicadores perinatais desfavoráveis ainda observados no país. Estratégias voltadas para as populações menos favorecidas socialmente são essenciais, visando a facilitar o ingresso precoce no pré-natal e o contato com os serviços de saúde para garantir a realização de cuidados efetivos em saúde. ${ }^{13}$

A condição da mulher é, portanto, de máxima importância para que o bebê tenha um desenvolvimento saudável, uma vez que um vínculo mãe-bebê desfavorável poderia facilitar a ocorrência de desordens ou perturbações na trajetória de desenvolvimento do bebê, assim como contribuir para a prevenção de problemas de saúde, emocionais e comportamentais do bebê. ${ }^{1}$

Durante o período investigado encontrou-se apenas uma criança nascida entre 22 a 27 semanas de gestação, 13 nascidas entre 28 a 36 semanas de gestação, sendo consideradas pré-termo. As crianças nascidas a termo (37 a 41 semanas) foram a maioria com 71,9\% (Tabela 6).

A morbilidade e a mortalidade perinatal estiveram sempre associadas com parto pré-termo, pós-termo e restrição de crescimento intrauterino. $\mathrm{O}$ conhecimento preciso da idade gestacional e da data provável do parto é, por isso, fundamental em Medicina Perinatal, para a correta orientação obstétrica e definição de estratégias assistenciais. ${ }^{20}$

Crianças que nascem no tempo ideal tendem a ter um desenvolvimento mais saudável do que as prematuras. A prematuridade é passível de prevenção se a causa for detectada precocemente, durante o pré-natal, por exemplo. O uso de drogas, diabetes, doença hipertensiva específica da gestação, infecções do trato urinário e outras doenças de base materna são fatores predisponentes 
à prematuridade e podem ser detectados $\mathrm{e}$ prevenidos durante o pré-natal. ${ }^{18}$

\section{CONSIDERAÇões FINAIS}

Obter informação sobre a característica de determinada população é a medida inicial para elaborar planos de intervenção fundamentados nas necessidades encontradas e tornar eficaz a assistência do serviço de saúde.

$\mathrm{O}$ perfil das gestantes atendidas no Centro de Saúde da Família do bairro Dom
Expedito Lopes é de mulheres jovens, com ensino médio, casadas e submetidas a parto cesáreo. Essas informações mostram a importância de campanhas educativas de orientação e convencimento, quanto às vantagens do parto normal.

É fundamental caracterizar o perfil sociodemográfico e obstétrico das gestantes, pois muitas das alterações físicas e emocionais da mãe são transmitidas para o feto. Portanto, a condição da mulher é de grande importância para que o bebê tenha um desenvolvimento saudável.

\section{REFERÊNCIAS}

1 Correia LL, Daniel JP. Perfil sociodemográfico e obstétrico de puérperas atendidas na Maternidade de um Hospital Universitário. ENEPEX, 2013. Disponível em: http://eventos.ufgd.edu.br/ enepex/anais/arquivos/286.pdf

2 Amorim MHC, Barbosa TKO, Bravim LR, Leite FMC, Primo CC. A influência das características socioeconômicas no perfil obstétrico de puérperas. Aquichan, 2014. Disponível em: www. scielo.org.co/pdf/aqui/v14n4/v14n4a11.pdf

3 Nicésio MG et al. Perfil de gestantes atendidas na atenção primária em uma cidade do interior de Minas Gerais. Rev Iniciação Cient Libertas. 2018, 8 (1).

4 Arcos E., Bonatti C., Muñoz LA, Sanchez X., Vollrath A. Vivenciando a maternidade em contextos de vulnerabilidade social: uma abordagem compreensiva da fenomenologia social. Rev Latino-Americana Enferm, 2013. Disponível em: www.scielo.br/pdf/rlae/v21n4/pt_01041169-rlae-21-04-0913.pdf

5 Pelloso SM, Silva GF. Perfil das parturientes e seus recém-nascidos atendidos em um hospital-escola do Noroeste do Estado do Paraná. Rev Escola Enferm USP, 2009. Disponível em: www. scielo.br/pdf/reeusp/v43n1/12.pdf

6 Dias EG et al. Perfil socioeconômico e gineco-obstétrico de gestantes de uma estratégia de Saúde da Família do norte de Minas gerais. Rev Saúde Desenvol. 2018, 12 (10): 284-297.

7 Oliveira MCR, Ximenes FMA. A influência da idade materna sobre as condições perinatais. Rev Bras Promoção Saúde, 2004. Disponível em: periodicos. Unifor.br/RBPS/article/download/677/2047

8 Ministério da Saúde (BR). Gestação de alto risco: manual técnico, 4. ed. Brasília (DF): O Ministério; 2000. Disponível em: http://bvsms.saude.gov.br/bvs/publicacoes/gestacao_alto_risco_ maual_tecnico_4ed.pdf

9 Araújo VS, Braga LS, Cabral ACF, Cordeiro CA, Dias MD, Moraes MN. Percepções da gravidez em adolescentes gestantes. Rev Pesquisa: Cuidado é Fundamental Online, 2015. Disponível em: www.ssoar.info/ssoar/bitstream/handle/document/54276/ssoar-revpesquisa- 2015-2-cabral_et_ al Perceptions_of pregnancy_in_pregnant.pdf?sequence $=1$ 
10 Jesus MP, Moraes FR, Motta M. Dificuldades e desafios do pré-natal sob a perspectiva das adolescentes grávidas. Adoles Saúde, 2017. Disponível em: www.adolescenciaesaude.com/detalhe_ artigo.asp?id=672

11 Barreto CN et al. Atenção pré-natal na voz das gestantes. Rev Enferm. 2013, 7 (5): 4.354-4.363.

12 Benetti BA, Fontoura LO, Hohendorff JV, Levandowski DC, Prati LE. Caracterização do Perfil Sócio-Demográfico de Gestantes Adolescentes: Revisão da Literatura Brasileira. Psychologica, 2009. Disponível em: impactum-journals.uc.pt/psychologica/article/view/983/432

13 Dias MAB, Domingues RMSM, Gama SGN, Leal MC, Theme-Filha MM, Torres JA, Viellas EF. Adequação da assistência pré-natal segundo as características maternas no Brasil. Rev Pan-americana Saúde Pública, 2015. Disponível em: www.scielosp.org/pdf/rpsp/2015.v37n3/140-147/pt

14 Fundo das Nações Unidas para a Infância (UNICEF). Quem Espera, Espera, 2017. Disponível em: www.unicef.org/brazil/pt/quem_espera_espera.pdf

15 Amorim AS, Fernandes MVM, Neto AUA, Oliveira MAS, Silva AHR. Perfil obstétrico de parturientes atendidas em um hospital público do interior do estado do Ceará, Brasil. Rev Enferm UFPI, 2015. Disponível em: www.ojs.ufpi.br/index.php/reufpi/article/view/4609/pdf

16 Silva HTFML, Tavares FM. Perfil Gestacional, Obstétrico e Neonatal na unidade de Terapia Intensiva de um Hospital Público do interior da Bahia. Rev. Saúde. com, 2017. Disponível em: periodicos2.uesb.br/index.php/rsc/article/download/3322/2762/

17 Duro SMS, Facchini LA, Fassa AG, Fernandes PAA, Fischer T., Nunes BP, Saes MO, Silveira DS, Siqueira FCV, Thume E., Tomasi E. Qualidade da atenção pré-natal na rede básica de saúde do Brasil: indicadores e desigualdades sociais. Cad Saúde Pública, 2017. Disponível em: www. scielo.br/pdf/csp/v33n3/1678-4464-csp-33-03-e00195815.pdf

18 Secretaria de Estado da Saúde de São Paulo. Atenção a Gestante e a Puérpera no SUS-SP. Manual Técnico do Pré-Natal e Puerpério, 2010. Disponível em: www.saude.sp.gov.br/resources/ses/ perfil/gestor/destaques/atencao-a-gestante-e-a-puerpera-no-sus-sp/manual-tecnico-do-pre-natal-e-puerperio/manual_tecnicoii.pdf

19 Calegari RS, Gonçalves AC, Gouveia HG. Intercorrências clínicas e obstétricas vivenciadas por mulheres no pré-natal. Cogitare Enferm, 2016. Disponível em: revistas.ufpr.br/cogitare/article/ view/44604/28558

20 Matias A, Montenegro N, Tiago P. Cálculo da Idade Gestacional Métodos e Problemas. Acta Médica Portuguesa, 2002. Disponível em: www.actamedicaportuguesa.com/revista/index.php/ amp/article/viewFile/1912/1480

\section{DAdos dos AUTORES}

Leonardo Sales Ribeiro Silva - Acadêmico do Curso de Medicina Centro. Universitário INTA - UNINTA; Bolsista de Iniciação Científica do Centro Universitário INTA - UNINTA. Sobral/ CE - Brasil. leo-145@hotmail.com

Ana Neiline Cavalcante. Mestre. Docente no curso de Medicina do Centro Universitário INTA - UNINTA. Sobral/CE - Brasil. ecobio@zipmail.com.br

Maria Auxiliadora Silva Oliveira. Bióloga. Mestre. Docente no Centro Universitário INTA UNINTA. Sobral/CE - Brasil. myresearchbio@hotmail.com

Submetido em: $24-4-2018$

Aceito em: 22-6-2020 